\title{
An Improved Predictive Functional Control with Minimum-Order Observer for Speed Control of Permanent Magnet Synchronous Motor
}

\author{
Shuang Wang ${ }^{\dagger}$, Junyong Fu*, Ying Yang* and Jian Shi*
}

\begin{abstract}
In this paper, an improved predictive functional control (PFC) scheme for permanent magnet synchronous motor (PMSM) control system is proposed, on account of the standard PFC method cannot provides a satisfying disturbance rejection performance in the case of strong disturbances. The PFC-based method is first introduced in the control design of speed loop, since the good tracking and robustness properties of the PFC heavily depend on the accuracy of the internal model of the plant. However, in orthodox design of prediction model based control method, disturbances are not considered in the prediction model as well as the control design. A minimumorder observer (MOO) is introduced to estimate the disturbances, which structure is simple and can be realized at a low computational load. This paper adopted the MOO to observe the load torque, and the observations are then fed back into PFC model to rebuild it when considering the influence of perturbation. Therefore, an improved PFC strategy with torque compensation, called the $\mathrm{PFC}+\mathrm{MOO}$ method, is presented. The validity of the proposed method was tested via simulation and experiments. Excellent results were obtained with respect to the speed trajectory tracking, stability, and disturbance rejection.
\end{abstract}

Keywords: Torque compensation, Disturbance observer, Minimum-order observer (MOO), Predictive functional control (PFC), Permanent magnet synchronous motor (PMSM).

\section{Introduction}

In recent decades, the permanent magnet synchronous motor (PMSM) has been generally replacing DC motors and induction motors in a largely of industrial applications, such as numerically controlled machine tools, electric vehicles and robotics. It is able to offer numerous advantages over the two previously mentioned motors, including a simple structure, high efficiency, high power density and high torque-to-current ratio. Despite its advantages, it is still challenging to control a PMSM to achieve good transient performance under all operating conditions. This is due to the fact that the PMSM is multivariable, nonlinear and strong coupled systems that are extremely sensitive to disturbances.

As a classical linear control scheme, the traditional proportional integral (PI) control is usually used to adjust the static and dynamic performance of a system. However, the control system of a PMSM makes it difficult to achieve a good control effect with the PI control scheme due to the presence of nonlinearity and uncertainty [1-3]. Scholars have put forward many advanced control methods to optimize the PMSM control system in recent years, e.g., adaptive control [4], fuzzy control [5], sliding mode

$\dagger$ Corresponding Author: School of Mechatronic Engineering and Automation, Shanghai University, China. (wang-shuang@shu.edu.cn)

* School of Mechatronic Engineering and Automation, Shanghai

University, China. (yangying $\mathrm{h} @ 163 . \mathrm{com})$

Received: June 6, 2016; Accepted: October 11, 2016 control [6], feedback linearization [7], genetic algorithm control [8], neural network control [9], disturbance observer based (DOB) [10-12], and so on. These approaches have been successfully applied to PMSM control systems, and can improve the control performance of a motor from different aspects.

Predictive control is a new type computer control algorithm that produced industrial process control field in the 1970s. It has an excellent control effect and is suitable for industrial production processes which are relatively complex and where it is difficult to establish accurate an digital model, because it uses a multi-step test, receding optimization, and feedback correction strategy [13, 14]. Model predictive control (MPC) is the most widely used method of predictive control. The core of the MPC is to replace a "closed loop optimal control" with an "open loop optimal control" in a time-varying region. As a practical alternative approach, MPC has received a great deal of attention. There are many acclaiming research reports on the application of the MPC method to PMSM control systems. The controllers developed in $[7,15,16]$, give good performance. However, the MPC control requires too much calculation, which makes this method difficult to apply to fast dynamic systems $[17,18]$.

To alleviate computational effort and reduce numerical problems, particularly in large prediction horizon, a simplified MPC method, i.e., the predictive functional control (PFC) method is employed as the adopted control scheme in this study. This method was presented by 
Richalet and Kuntze in 1986 [19, 20]. PFC which enhances the advantages of this method, such as feed-forwarding, constraints handling, no-lag error on the dynamic set points, is an easy trade-off between robustness and dynamic specifications. It not only retains the advantages of on-line optimization and constraint processing of the MPC, but also has a lower online computational burden, which can achieve a simpler and more intuitive design criteria combined with a higher control precision [21].

In practical industrial applications, disturbances always exists, it is noted that the standard PFC method does not achieve a satisfactory effect in the presence of strong disturbances. Because PFC assumes that all un-modeled signals remain the same with prediction current errors, which makes it hard to counteract steady-state errors when meeting interference or inaccurate model, especially in the presence of strong interference conditions, which may cause deterioration of the performance of the closed-loop control, it is difficult for the prediction model to precisely predict future outputs [22]. As a result, the future control action obtained by optimizing a certain performance index cannot react well to resist disturbances. In [23], the scholars introduce an extended state observer (ESO) to estimate the lumped disturbances and add a feedforward compensation $q$-axis current item based on the estimated disturbances. This strategy can reduce prediction errors and improve the performance of drive control system. In [24], the authors designed a fuzzy sliding mode speed controller with a load torque observer, which can effectively mitigate chattering and guarantee robust speed control of a PMSM under model parameter and load torque variations. References $[25,26]$ utilize full-order observers to against stator resistance and inductance variations for the sensorless motor drive. The above researches focused on external disturbance influence on the system, but they both have a complex structure and high computational load.

To overcome the above-mentioned drawbacks and improve the disturbance rejection performance of the PFC scheme, this paper proposed an improved PFC method, called the PFC+ minimum-order observer (MOO) method, is proposed in this paper. Here, we introduce a simple state observer, which is the minimum-order of Luenberger-style observer to estimate the motor load torque disturbance. In 1964, Luenberger proposed state observer design theoretical for linear systems, has received extensive discussion [27-29]. Reference [30] has proposed a special angle and speed state observer to modify the model predictive direct current control strategy in order to obtain high dynamics. A model-based robust controller is used in [31] to estimate the load torque, and analyzed the convergence of the observer estimation error, the simulation and experimental results are presented to verify that the proposed methodology achieves a better robust performance. In [32], A reduced-order rotor flux is used to estimate the position of the rotor flux and hence the rotor speed, excellent results verified the performances of the observer. Meanwhile, the minimum-order observer (MOO) has been well studied and applied successively in [33, 34]. The MOO has some attractive features, e.g., 1), its order is the minimum second. 2), observer gain is a simple constant and pole allocation can be easily designed. 3), it utilizes motor parameters in a very simple manner. 4), its structure is very simple, and it can be realized at a very-low computational load. Based on this, a practical MOO is constructed to obtain information on the load torque. Then the PFC controller is compensated with observed values, and the PFC model is reconstructed, MOO is utilized to reduce the prediction error in PFC algorithm and cope with disturbances. Finally, the improved PFC method proposed in this paper was verified by the simulation and experiment, the results verify its effectiveness.

This paper is organized as follows. In section 2, the PMSM model is presented. Section 3 presents the design details of a speed controller using the PFC method. The studied PFC with MOO strategy is developed in Section 4 for the speed loop. Section 5 gives the simulation and the experimental results of the predictive control scheme for the PMSM control system. Section 6 concludes the paper.

\section{Mathematical Model of the PMSM}

The mathematical model of a surface mounted PMSM in the rotor reference frame can be expressed in the bilinear form as

$$
\frac{d}{d t}\left[\begin{array}{c}
i_{d} \\
i_{q} \\
\omega
\end{array}\right]=\left[\begin{array}{ccc}
-R_{s} / L_{a} & n_{p} \omega & 0 \\
-n_{p} \omega & -R_{s} / L_{a} & n_{p} \psi_{f} / L_{a} \\
0 & K_{t} / J & -B / J
\end{array}\right]\left[\begin{array}{c}
i_{d} \\
i_{q} \\
\omega
\end{array}\right]+\left[\begin{array}{c}
u_{d} / L_{a} \\
u_{q} / L_{a} \\
-T_{L} / J
\end{array}\right]
$$

where, $u_{d}$ and $u_{q}$ are respectively the $d$-axis and $q$-axis components of the armature voltage, $i_{d}$ and $i_{q}$ are respectively the $d$-axis and $q$-axis components of the armature current, $R_{s}$ is the stator resistance, $L_{a}$ is the stator inductance, $L_{d}$ and $L_{q}$ are the inductances of the $d-q$ axes satisfying $L_{d}=L_{q}=L_{a}, n_{p}$ is the number of pole pairs, $\psi_{f}$ is the rotor of the permanent magnet flux linkage, $\omega$ is the rotor mechanical velocity, $T_{L}$ is the motor load torque, $J$ is the moment of inertia, and $B$ is the coefficient of the friction.

The field oriented control (FOC) strategy is employed for PMSM. Under this scheme, the automatic speed regulator (ASR) and automatic current regulator (ACR) are cascaded, and the d-axis reference current is usually set to be zero. Then the electromagnetic torque is given as

$$
T_{e}=1.5 n_{p} \psi_{f} i_{q}=K_{t} i_{q}
$$

where, $T_{e}$ is the electromagnetic torque.

The mechanical equation of a dynamic model for a 


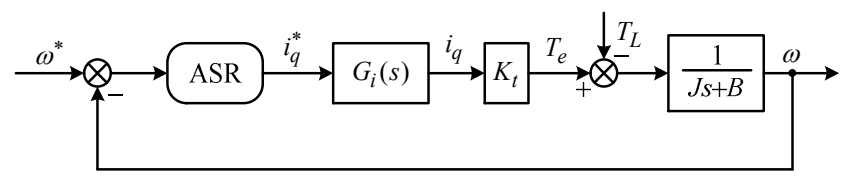

Fig. 1. Block diagram of the simplified PMSM control system.

motor is

$$
\omega(s)=\frac{K_{t} i_{q}^{*}(s)-T_{L}(s)}{J_{S}+B}
$$

Remark 1: In the following, the symbol " $s$ " is used as a differential operator $d / d t$ or as a Laplace operator with no comment as long as no confusion occurs.

The implementation of the predictive functional control strategy depends on an appropriate model. The dynamic model of motor is a nonlinear and strong coupling system, because the current band width is far greater than the speed loop bandwidth. The setting time of the q-axis current (iq) is far less than the setting time of speed, as shown in Fig. 9-11.Then the current loop can be assumed to be ignored and transfer function is reduced to $G_{i}(s) \approx 1$. The simplified block diagram of a PMSM is shown in Fig. 1.

\section{PFC Controller Design for the PMSM}

In practical applications, PFC restructures the control inputs by considering each moment of the control inputs as a linear combination of several pre-selected basic functions, and then obtaining a linear weighting coefficient through the online optimization, and finally getting the control input of the future [23]. In this paper, the PFC design process is as follows.

\subsection{Base functions}

The controlled variable can be expressed as a linear combination of several known base functions in the PFC control strategy. It is related to the plant characteristic and the tracking set value,

$$
u(k+i)=\sum_{j=1}^{N} \mu_{j} f_{j}(i)
$$

where, $u(k+i)$ is the system output at time $(k+i)$. The choice of base functions depends on the characteristics of the controlled plant and the set values, such as a step, ramp and exponential function. The desirable output control variable of the PFC is the reference current $i_{q}^{*}$. Therefore:

$$
i_{q}^{*}(k+i)=\sum_{j=1}^{N} \mu_{j} f_{j}(i)
$$

where, $f_{j}(i)$ is the value of the base functions at time $t=i T_{s}, T_{s}$ is the sampling period, $i=1,2, \cdots h, h$ is the predictive optimal length, and $\mu_{j}$ is the linear combination coefficient. Because the choice of basic functions does not directly affect the stability or the dynamic state of a closedloop system, this system chooses a step function as the base functions, Set $N=1, f_{1}(i)=1$. Therefore:

$$
i_{q}^{*}(k)=\mu_{1}
$$

\subsection{Prediction model}

A Prediction model is used to predict the output of a process in a future period of time according to historical information and the current input of objects studied. Generally, disturbances are not considered in the prediction model as well as the control design, Eq. (3) can be written in the form of difference equation after zero-order holder discrete sampling:

$$
\omega_{m}(k+1)=\alpha_{m} \omega_{m}(k)+K_{m} i_{q}^{*}(k)
$$

where, $\alpha_{m}=e^{-T_{S} B / J}$, and $K_{m}=\left(1-\alpha_{m}\right) K_{t} / B$ are both the coefficients of a prediction model equation. $\omega_{m}(k+1)$ is the speed prediction value at time $(k+1)$.

However, the influence of disturbances is not considered in the prediction model which degrades the prediction precision in the presence of strong disturbances, to improve the disturbance rejection ability of system, an improved PFC method by embedding the estimated value of load torque disturbance information into the prediction model, where disturbance information is obtained by a minimumorder observer (MOO) (The studied of MOO strategy is developed in Section 4). Considering the load torque disturbance information $\hat{T}_{L}$, it follows from (3) and (7) that is given as

$$
\omega_{m}(k+1)=\alpha_{m} \omega_{m}(k)+K_{m} i_{q}^{*}(k)+K_{m} \hat{T}_{L}(k) / K_{t}
$$

In the next sampling time $(k+2)$,

$$
\begin{gathered}
\omega_{m}(k+2)=\alpha_{m} \omega_{m}(k+1)+K_{m} i_{q}^{*}(k+1) \\
+K_{m} \hat{T}_{L}(k+1) / K_{t}
\end{gathered}
$$

According to the mean-level control method, assuming that the values of the control variables are a constant in the future, $i_{q}^{*}(k)=i_{q}^{*}(k+1)=\cdots=i_{q}^{*}(k+h-1)$. Substituting (8) into (9) yields:

$$
\begin{aligned}
\omega_{m}(k+2)= & \alpha_{m}^{2} \omega_{m}(k)+K_{m}\left(1+\alpha_{m}\right) i_{q}^{*}(k) \\
& +K_{m}\left(1+\alpha_{m}\right) \hat{T}_{L}(k) / K_{t}
\end{aligned}
$$

Followed by the superposition, the output of the 
prediction model can be obtained as:

$$
\begin{aligned}
\omega_{m}(k+i)= & \alpha_{m}^{i} \omega_{m}(k)+K_{m}\left(1+\alpha_{m}+\cdots+\alpha_{m}^{i-1}\right) i_{q}^{*}(k) \\
& +K_{m}\left(1+\alpha_{m}+\cdots+\alpha_{m}^{i-1}\right) \hat{T}_{L}(k) / K_{t}
\end{aligned}
$$

Eq. (11) can be written in a matrix form as:

$$
W_{m}(k)=W_{o}(k)+W_{b}(k)\left[\mu_{1}(k)+\hat{T}_{L}(k) / K_{t}\right]
$$

where

$$
\begin{aligned}
& W_{m}(k)_{(h \times 1)}=\left[\omega_{m}(k+1) \cdots \omega_{m}(k+h)\right]^{T} \\
& W_{o}(k)_{(h \times 1)}=\left[\alpha_{m} \cdots \alpha_{m}^{h}\right]^{T} \omega_{m}(k) \\
& W_{b}(k)_{(h \times 1)}=\left[K_{m} \cdots K_{m}\left(1+\cdots+\alpha_{m}^{h-1}\right)\right]^{T}
\end{aligned}
$$

\subsection{Error correction}

Considering model adaptation, parameter detuning and interference, the actual output and the predicted output usually contain certain errors. System error during the prediction time domain can be considered to be the same as the error at this time.

$$
e(k+i)=\cdots=e(k+1)=e(k)=\omega(k)-\omega_{m}(k)
$$

where, $\omega(k)$ is the practical system output measured at time $k$.

\subsection{Reference trajectory}

For a stable system, a first-order exponential form is usually used. Accordingly the reference trajectory equation is as follows:

$$
\omega_{r}(k+i)=\omega^{*}(k+i)-\alpha_{r}^{i}\left[\omega^{*}(k)-\omega(k)\right]
$$

where, $\omega^{*}$ is the given speed, $\omega_{r}$ is the reference trajectory of the speed which is the expected following value of the actual system after the softening treatment according to the expected trajectory. The softness factor $\alpha_{r}^{i}=e^{\left(-T_{S} / T_{r}\right)}, T_{r}$ is the expected system response time.

\subsection{Receding optimization}

Receding optimization is through the optimization of a performance index to determine a control action of the future. Performance indicators are generally taken as the minimum variance between the object output at a certain time in the future and the desired trajectory at this moment. The quadratic performance index set of the cost function for the PMSM speed control is expressed as:

$$
\min \mathfrak{I}=\sum_{i=1}^{h}\left[\omega_{r}(k+i)-\omega_{m}(k+i)-e(k+i)\right]^{2}+r^{2} i_{q}^{* 2}(k)
$$

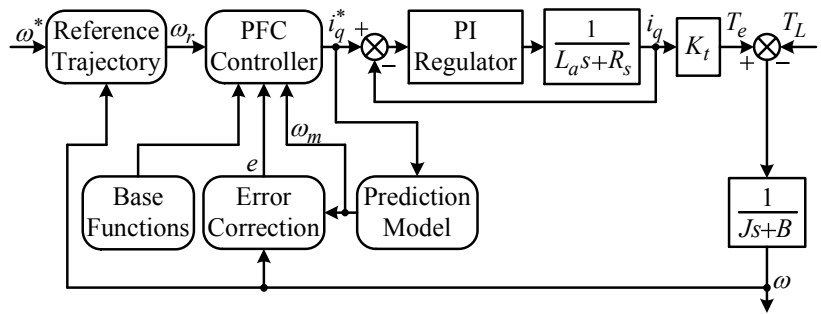

Fig. 2. Block diagram of the simplified PMSM control system using PFC method.

The equation can be written in another form of norm:

$$
\mathfrak{J}=\left\|W_{r}(k)-W_{m}(k)-E(k)\right\|^{2} Q_{q}+R_{r} i_{q}^{* 2}(k)
$$

where

$$
\begin{aligned}
& W_{r}(k)_{(h \times 1)}=\left[\omega_{r}(k+1) \cdots \omega_{r}(k+h)\right]^{T} \\
& E(k)_{(h \times 1)}=[\mathrm{e}(k+1) \cdots e(k+h)]^{T} \\
& Q_{q}(k)_{(h \times h)}=\operatorname{diag}\left[q_{1}^{2}, \cdots, q_{h}^{2}\right]
\end{aligned}
$$

$Q_{q}(k)_{(h \times h)}$ is the weighted coefficient of controlled variables, and $R_{r(1 \times 1)}=\left[r^{2}\right]$ is weighted coefficient of the input. Since the mechanical model of the motor is linear characteristic, it can determine the minimum value of the cost function $\mathfrak{I}$ and the real-time update coefficient matrix at each sampling time. Let $\partial \mathfrak{I} / \partial i_{q}^{*}=0$, and the controlled variable of $i_{q}^{*}$ can be obtained.

$$
\begin{aligned}
i_{q}^{*}= & \left(W_{b}^{T} Q_{q} W_{b}+R_{r}\right)^{-1} W_{b}^{T} Q_{q}\left[W_{r}(k)-W_{o}(k)\right. \\
& \left.-E(k)-W_{b} \hat{T}_{L}(k) / K_{t}\right]
\end{aligned}
$$

The block diagram of the simplified PMSM control system is shown in Fig. 2. According to the predictive function control algorithm, when combined with the vector control strategy of the PMSM control system, the AC control system with the PFC-PI cascade control is designed, using the PFC controller to replace the traditional automatic speed regulator.

\section{PFC Design Using a Minimum-Order Observer}

For the foregoing reasons, the influence of disturbances is not considered in the prediction model, and PFC controller lacks an external disturbance compensation term in the process of modeling, resulting in a degradation of the control performance in the dynamic processes of addition and subtraction of loads [22]. In order to work out this problem, we introduce state observer to estimate the disturbances. Here, a minimum-order observer is only designed to observe torque disturbance, then feedback 
the observations to PFC controller. This can reconstruct the predictive model, and implement improvement over the original PFC controller, which improves the robustness against disturbance.

Refer to (1), the mechanical equation of PMSM, disturbance torque is extended to a state variable, load torque cannot be derived from the formula, but the changing rate of the load torque controller is much lower than sampling frequency, so it can be hypothesized that $d T_{L} / d t=0$. There is a linear relationship between the rotor position and rotor velocity, $\omega=s \theta$. This paper selects the state variable, $x=\left[\begin{array}{ll}\omega & T_{L}\end{array}\right]^{T}$, the output variable $y=\omega$, and the Eq. (1) can be written in another form as:

$$
\frac{d}{d t}\left[\begin{array}{l}
\omega \\
T_{L}
\end{array}\right]=\left[\begin{array}{cc}
-B / J & -1 / J \\
0 & 0
\end{array}\right]\left[\begin{array}{l}
\omega \\
T_{L}
\end{array}\right]+\left[\begin{array}{c}
1 / J \\
0
\end{array}\right] T_{e}
$$

Let us define, $A=\left[\begin{array}{cc}-B / J & -1 / J \\ 0 & 0\end{array}\right], C=\left[\begin{array}{c}1 / J \\ 0\end{array}\right], D=\left[\begin{array}{ll}1 & 0\end{array}\right]$, $u=T_{e}$, it follows from (18) that the linear state equation and output equation of the control system can then be obtained as:

$$
\left\{\begin{array}{l}
\dot{x}=A x+C u \\
y=D x
\end{array}\right.
$$

Fig. 3 is a block diagram of a state observer, which is fully based on the state equation of (19), the state observer expression can be derived as [33]:

$$
\left\{\begin{array}{l}
\dot{\hat{x}}=A \hat{x}+C u+\lambda(y-\hat{y}) \\
\hat{y}=D \hat{x}
\end{array}\right.
$$

where, $\hat{x}$ is the estimated state value, $\hat{x}=\left[\begin{array}{ll}\hat{\omega} & \hat{T}_{L}\end{array}\right]^{T}, \lambda$ is the feedback gain matrix, $\lambda=\left[\begin{array}{ll}\lambda_{1} & \lambda_{2}\end{array}\right]^{T}$. So, the Eq. (20) can be written in the form as

$\frac{d}{d t}\left[\begin{array}{l}\hat{\omega} \\ \hat{T}_{L}\end{array}\right]=\left[\begin{array}{cc}-B / J & -1 / J \\ 0 & 0\end{array}\right]\left[\begin{array}{l}\hat{\omega} \\ \hat{T}_{L}\end{array}\right]+\left[\begin{array}{c}1 / J \\ 0\end{array}\right] T_{e}+\left[\begin{array}{l}\lambda_{1} \\ \lambda_{2}\end{array}\right](\omega-\hat{\omega})$

From (18)-(21), the equations for the state error

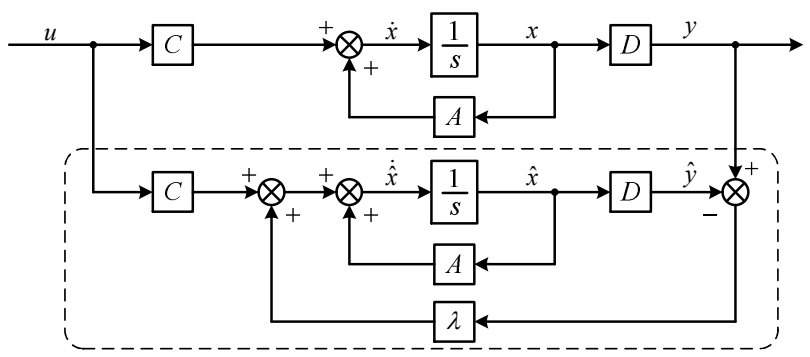

Fig. 3. Block diagram of the state observer. $\hat{e}=x-\hat{x}$, is given by $(22)$

$$
\frac{d}{d t}[\hat{e}]=(A-\lambda D) \hat{e}=\left[\begin{array}{cc}
-\frac{B}{J}-\lambda_{1} & -\frac{1}{J} \\
-\lambda_{2} & 0
\end{array}\right] \hat{e}
$$

On the basis of (22), the characteristic equation as follows

$$
\begin{aligned}
\operatorname{det}[s I-(A-\lambda D)] & =\left|s\left[\begin{array}{ll}
1 & 0 \\
0 & 1
\end{array}\right]-\left[\begin{array}{cc}
-\frac{B}{J}-\lambda_{1} & -\frac{1}{J} \\
-\lambda_{2} & 0
\end{array}\right]\right| \\
& =s^{2}+\left(\lambda_{1}+\frac{B}{J}\right) s-\frac{\lambda_{2}}{J}=0
\end{aligned}
$$

The dynamic characteristics of state error $\hat{e}$ entirely depends on the eigenvalues of $A-\lambda D$, so, to select suitable eigenvalues of the observer can makes error $\hat{e}$ converges to zero as soon as possible, which simplifies the characteristic polynomial to

$$
s^{2}-(\alpha+\beta) s+\alpha \beta=0
$$

Coefficients $\alpha$ and $\beta$ are desired poles of design parameters, if the friction coefficient $B$ is ignored, from (23)and (24), the feedback gain can obtained as

$$
\left\{\begin{array}{l}
\lambda_{1}=-(\alpha+\beta) \\
\lambda_{2}=\alpha \beta J
\end{array}\right.
$$

Performance conditions: All of desired poles have negative real part is a convergence condition of state observer error equation $\hat{e}$, i.e., all the poles must be located in negative half part of s-plane. Meanwhile, pole position the farther from imaginary axis, the greater value of the feedback gain matrix $\lambda$, and the faster dynamic response of the observed variables also.

According to the Laplace transform, Eq. (21) can be rewritten as (26) and (27).

$$
\begin{gathered}
\hat{\omega}=\frac{T_{e}+\left(\lambda_{1} J-\frac{\lambda_{2}}{s}\right)(\omega-\hat{\omega})}{J_{S}+B} \\
\hat{T}_{L}=\frac{\lambda_{2}}{s}(\omega-\hat{\omega})
\end{gathered}
$$

By substituting (26) into (27), the estimated load torque disturbance information of the minimum-order observer is expressed as:

$$
\hat{T}_{L}=\frac{\frac{\lambda_{2}}{s}\left[(J S+B) \omega-T_{e}\right]}{\left(\lambda_{1}+s\right) J+B-\frac{\lambda_{2}}{s}}
$$


Eq. (28) can be written in the form of difference equation after zero-order holder discrete sampling:

$$
\begin{aligned}
\hat{T}_{L}(k)= & {\left[\left(K_{1}+K_{2}-K_{3}\right) \hat{T}_{L}(k-1)-K_{2} \hat{T}_{L}(k-2)-K_{3} T_{e}(k)\right.} \\
& \left.-K_{2} K_{3} \omega(k-1)+\left(K_{2} K_{3}+K_{3} B\right) \omega(k)\right] / K_{1}
\end{aligned}
$$

where, $K_{I}=J \lambda_{1}+J / T s+B-\lambda_{2} T s, K_{2}=J / T s, K_{3}=\lambda_{2} T s$ are both the coefficients of equation.

Refer to (29), when substituting the discretized $\hat{T}_{L}(k)$ into (8) of the prediction model, an improved PFC strategy with torque compensation is implemented. The constructed minimum-order load torque observer is shown in Fig. 4.

Obviously, that the load torque observer has the following attractive features.

1) Its order is the minimum second.

2) Minimum order observer gain is a simple constant and pole allocation can be easily designed.

3 ) It utilizes motor parameters in a very simple manner.

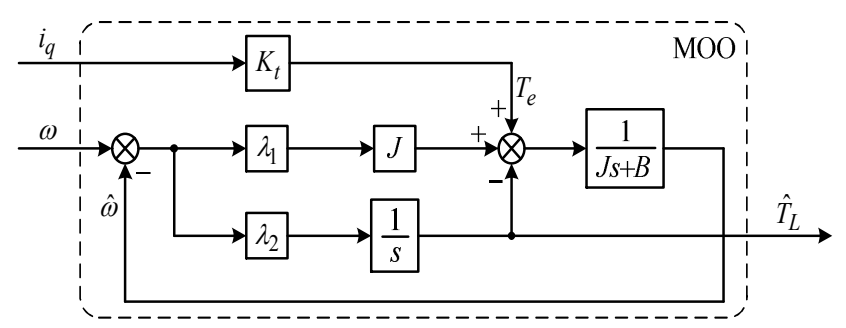

Fig. 4. Block diagram of the minimum-order observer.

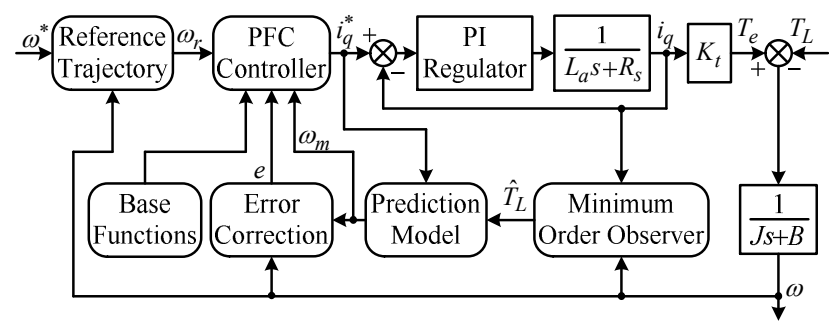

Fig. 5. Block diagram of the simplified PMSM control system using improved PFC with minimum-order observer.

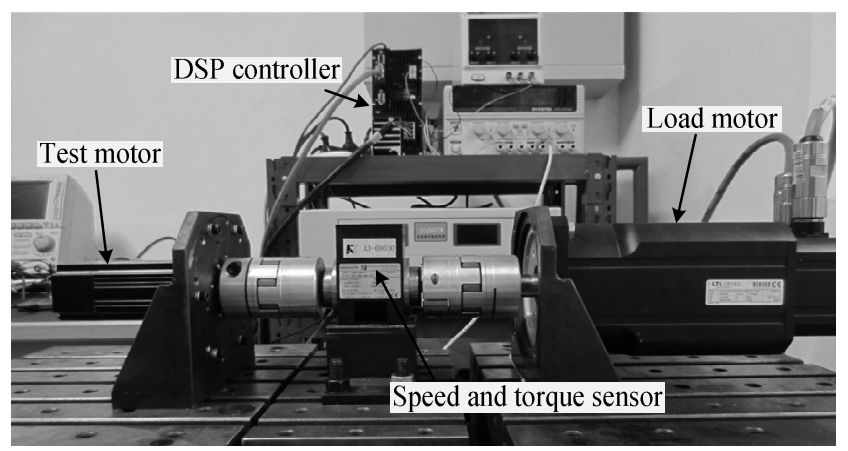

Fig. 6. Experimental setup.
4) Its structure is very simple, and it can be realized at a very-low computational load.

The overall structure of the proposed system consisting of a minimum-order observer and a PFC regulator, the block diagram of the PMSM control system with PFC and minimum order observer is shown in Fig. 6. Block diagram of the simplified PMSM control system using PFC method with minimum-order observer, as shown in Fig. 5.

\section{Simulation and Experimental Results}

In the processes of simulation and experiment, the parameters of the motor are listed in Table 1. Computer simulations have been carried out utilize Matlab/Simulink software package to verify performance and effectiveness of the proposed method. The performance of the methodology designs were compared using a simulation model built using the aforementioned motor model, inverse Park and Clarke transformations, and the discrete time control structure presented in this paper.

The experiment platform of driving control system has been built to evaluate the performance of the proposed improved PFC method. It takes Infineon's XMC4500 chip based on the ARM ${ }^{\circledR}$ Cortex as the core, with a CPU frequency of $120 \mathrm{MHz}$. The control algorithms use SVPWM. As shown in Fig. 6, it mainly includes two parts, the measured control system and the load system. The control system is driven by a three-phase pulse width modulation (PWM) inverter with an intelligent power module with a switching frequency of $10 \mathrm{kHz}$. The phase currents are measured by Hall-effect devices. The rotor position detection component uses an incremental photoelectric encoder of 2500 lines. The load motor and its controller choose Servo One industrial servo products of LUST,

Table 1. Parameters of the PMSM

\begin{tabular}{ccc}
\hline Parameter & Value & Unit \\
\hline Rated power & 400 & $\mathrm{~W}$ \\
Rated current & 1.9 & $\mathrm{~A}$ \\
Rated speed & 2000 & $\mathrm{rpm}$ \\
Rated torque & 1.21 & $\mathrm{~N} \cdot \mathrm{m}$ \\
Stator resistance & 5.8 & $\Omega$ \\
D-axis and Q-axis inductances & 0.0379 & $\mathrm{H}$ \\
Rotor flux linkage & 0.3675 & $\mathrm{~Wb}$ \\
Number of pole pairs & 4 & $/$ \\
Moment of inertia & $0.032 \times 10^{-3}$ & $\mathrm{~kg} \cdot \mathrm{m}^{2}$ \\
Coefficient of friction & $0.128 \times 10^{-3}$ & $\mathrm{~N} \cdot \mathrm{m} \cdot \mathrm{s} / \mathrm{rad})$ \\
\hline
\end{tabular}

Table 2. Comparison of performance indices (Simulation)

\begin{tabular}{ccccc}
\hline \multirow{2}{*}{$\begin{array}{c}\text { Reference } \\
\text { Speed }\end{array}$} & \multirow{2}{*}{ Indices } & \multicolumn{3}{c}{ Control Scheme } \\
\cline { 3 - 5 } & & PI & PFC & PFC+MOO \\
\hline \multirow{2}{*}{$500(\mathrm{rpm})$} & OS(\%) & 4.6 & 2.8 & 0 \\
& $t_{s}(\mathrm{~ms})$ & 130 & 117 & 95 \\
\hline \multirow{2}{*}{$2000(\mathrm{rpm})$} & OS $(\%)$ & 2.5 & 1.3 & 0 \\
& $t_{s}(\mathrm{~ms})$ & 270 & 244 & 239 \\
\hline
\end{tabular}


where the rated power of the motor is $3 \mathrm{~kW}$. The rotor position sensor uses a 24-bit multi ring absolute encoder from HEIDENHAIN.

The simulation and experimental analysis for the PI controller, PFC controller, and PFC controller+minimumorder observer (MOO) are illustrated in Figs. 7-16, Table 2, and Table 3 for four different scenarios: trajectory tracking performance evaluation under different speed, torque observe performance evaluation of the MOO, robustness of the proposed method against the inaccurate electrical parameters, and performance testing during an abrupt change of the load torque. They are $A, B, C$, and $D$,

Table 3. Comparison of performance indices (Experimental)

\begin{tabular}{ccccc}
\hline \multirow{2}{*}{$\begin{array}{c}\text { Reference } \\
\text { Speed }\end{array}$} & \multirow{2}{*}{ Indices } & \multicolumn{3}{c}{ Control Scheme } \\
\cline { 3 - 5 } & & PI & PFC & PFC+MOO \\
\hline \multirow{2}{*}{$500(\mathrm{rpm})$} & OS $(\%)$ & 6 & 4.08 & 1.2 \\
& $t_{s}(\mathrm{~ms})$ & 133 & 122 & 98 \\
\hline \multirow{2}{*}{$2000(\mathrm{rpm})$} & OS $(\%)$ & 2.45 & 1.5 & 0.35 \\
& $t_{s}(\mathrm{~ms})$ & 283 & 235 & 230 \\
\hline
\end{tabular}

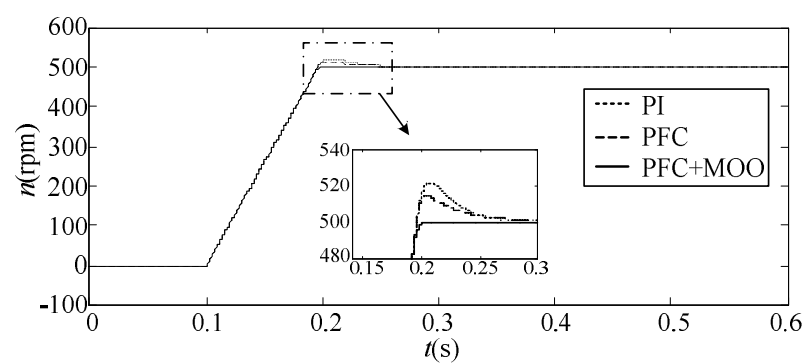

Fig. 7. Simulation response curves in case of $500 \mathrm{rpm}$ speed trajectory tracking for PI, PFC, and PFC + minimum-order observer(MOO).

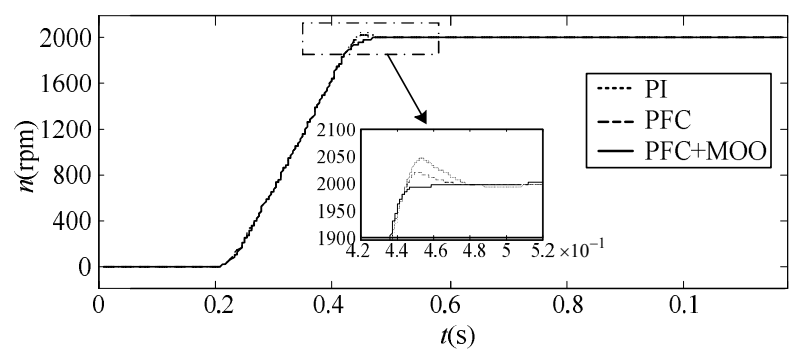

Fig. 8. Simulation response curves in case of $2000 \mathrm{rpm}$ speed trajectory tracking for PI, PFC, and PFC + MOO.

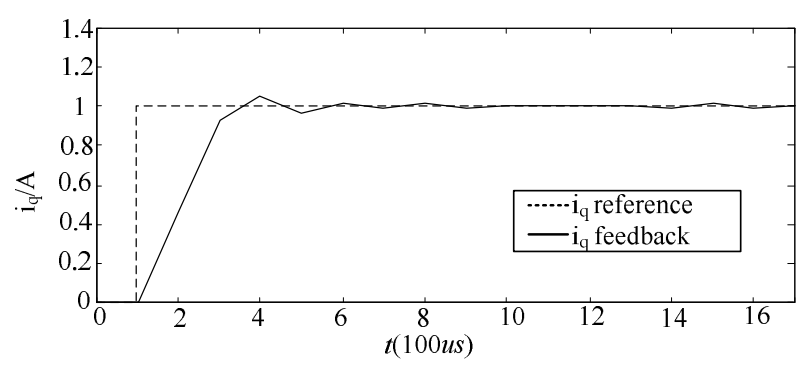

Fig. 9. The q-axis current (iq) instantaneously increases from 0 to $1 \mathrm{~A}$ (Experimental). respectively, in the followings.

\subsection{Trajectory tracking performance evaluation under different speed}

To evaluation the performance of presented three control algorithms, the speed trajectory tracking performance is investigated under three control methods. Figs. 7-10 shows the speed response waveforms under several given values respectively, then the no-load speed response was detected respectively in PI controller, PFC controller and PFC controller+MOO. Meanwhile, for a fair comparison, let the control inputs of three algorithms have the same condition limits and let both closed-loop systems achieve relatively good performances by regulating the parameters.

\subsubsection{Simulation conditions}

The parameters for the PI speed controller are: $K_{p}=0.016$, $K_{i}=0.02$, for the PI regulators of both current loops they are: $K_{p}=39, K_{i}=2400$, for the PFC controller they are: $\alpha_{m}=0.9548, \alpha_{r}=0.0183, K_{m}=1550$, PFC control

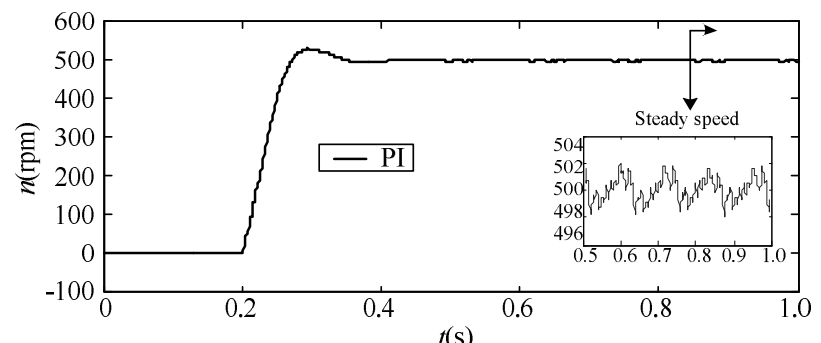

(a)

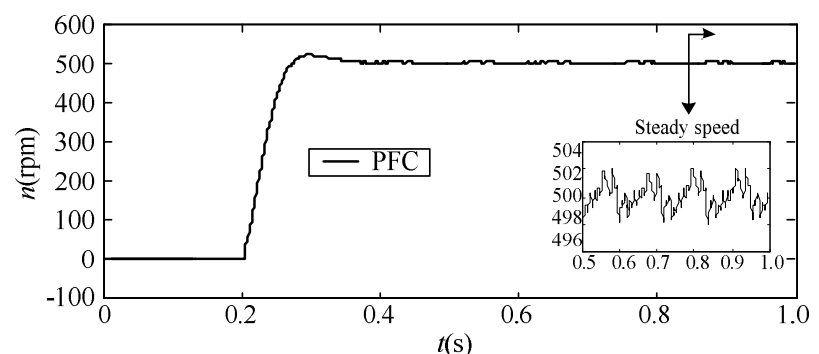

(b)

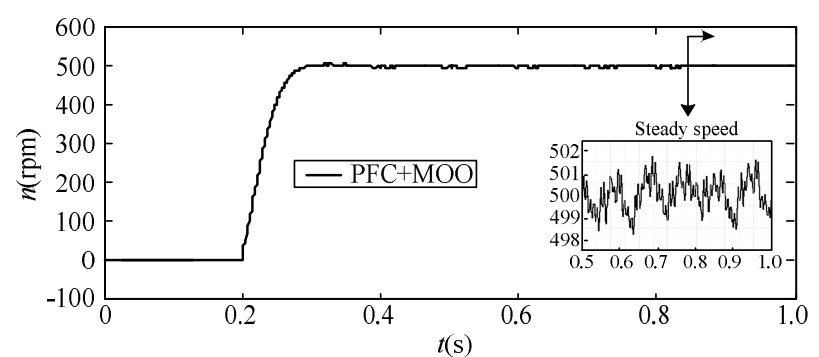

(c)

Fig. 10. Experimental response curves in case of $500 \mathrm{rpm}$ speed trajectory tracking: (a) PI; (b) PFC; (c) PFC $+\mathrm{MOO}$ 


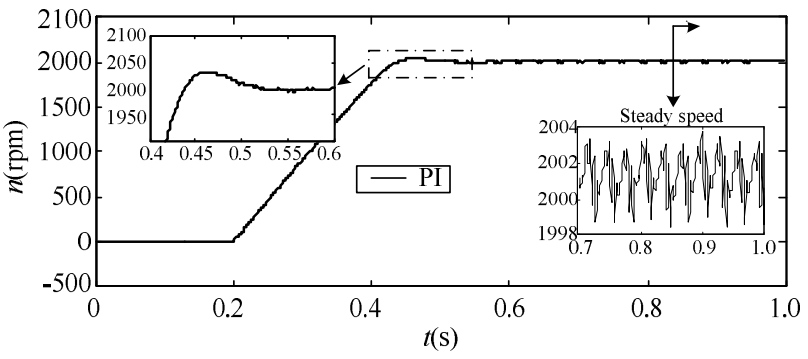

(a)

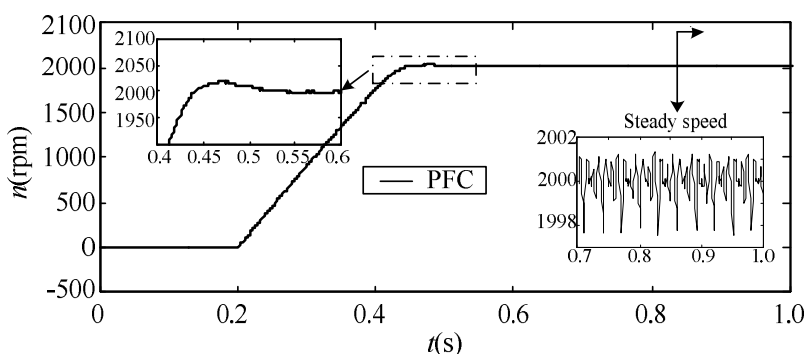

(b)

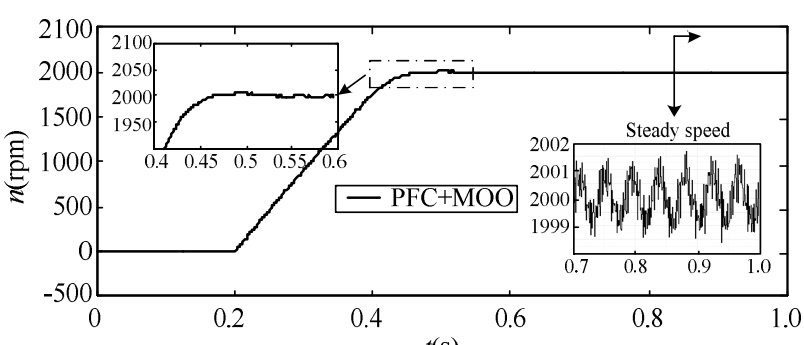

(c)

Fig. 11. Experimental response curves in case of $2000 \mathrm{rpm}$ speed trajectory tracking. a PI. b PFC. c PFC + MOO.

sampling period ${ }_{T s}=0.2 \mathrm{~ms} T_{s}=0.2 \mathrm{~ms}$, and for the reference trajectory expected closed-loop time $T_{r}=0.05 \mathrm{~ms}$.

Fig. 7 and Fig. 8 compared the speed tracking performance of the three algorithms. The starting speed is given as 500rpm and 2000rpm, respectively. Apparently, when applying the PFC+MOO strategy to simulation under the same conditions, speed of dynamic response process are smooth and steady, there are nearly no overshoot, and the raising speed regulate time obvious shorten. The simulation performance indexes of three control methods under different speed are shown in Table 2.

\subsubsection{Experimental conditions}

The parameters for the PI speed controller are: $K_{p}=18.5, K_{i}=1.5$, for the PI regulators of both current loops they are: $K_{p}=12, K_{i}=1500$, for the PFC controller they are: $\alpha_{m}=0.9548, \alpha_{r}=0.0183, K_{m}=1550$, PFC control sampling period $T_{s}=0.3 \mathrm{~ms}$, and for the reference trajectory expected closed-loop time $T_{r}=0.05 \mathrm{~ms}$.

Fig. 9 shows the experimental results for the PI current control method when the desired q-axis current (iq)

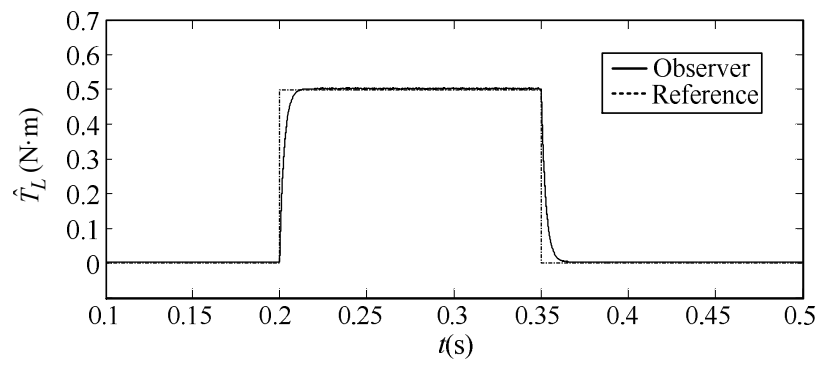

(a)

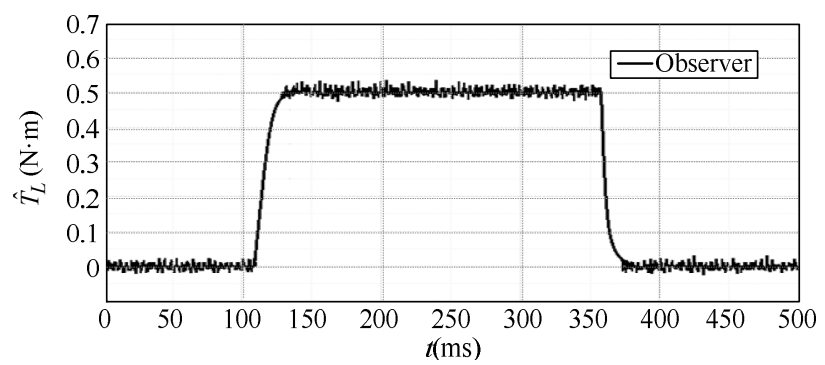

(b)

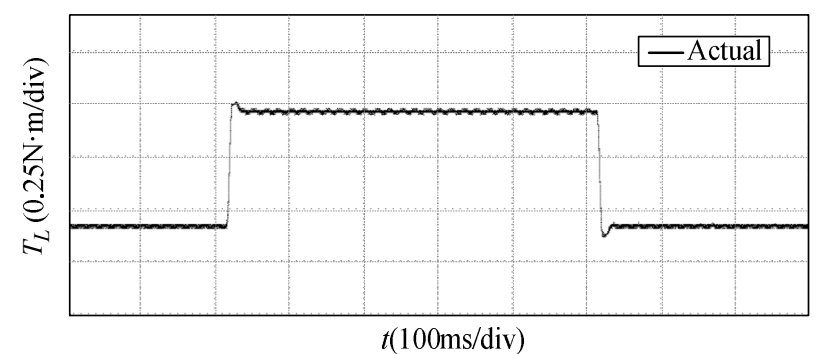

(c)

Fig. 12. Response curves of the minimum-order load torque observer: (a) Observer (Simulation); (b) Observer (Experimental); (c) Actual (Experimental).

instantaneously increases from 0 to $1 \mathrm{~A}$. The $i q$ rise time is three control periods $(300 u s)$. Fig. 10 and Fig. 11 compared the speed tracking performance of the three algorithms. Compared with other two controllers, the experimental results show that the improved PFC control system has better performance in both dynamic and steady state, it shows smallest overshoot, shortest settling time. The experimental performance indexes of three control methods under different speed are shown in Table 3.

\subsection{Torque observe performance evaluation of the MOO}

To ensure the MOO has a good dynamic response and observation precision, it is necessary to determine the values of feedback gain matrix $\lambda$, after a repeated simulation comparison, the desired poles of design parameters are $\alpha=-100, \beta=-100$. Refer to (25), $\lambda=\left[\begin{array}{l}\lambda_{1} \\ \lambda_{2}\end{array}\right]=\left[\begin{array}{c}-(\alpha+\beta) \\ \alpha \beta J\end{array}\right]$ $=\left[\begin{array}{c}200 \\ 10000 J\end{array}\right]$. 
A simulation and experimental comparison results are shown in Fig. 12. The load torque was abrupt change at a constant speed of $300 \mathrm{rpm}$, given the torque change range from $0 \mathrm{~N} \cdot \mathrm{m} \rightarrow 0.5 \mathrm{~N} \cdot \mathrm{m} \rightarrow 0 \mathrm{~N} \cdot \mathrm{m}$ under different time. Fig. 12(a) is a simulation load torque observe curves of $\hat{T}_{L}$, Fig. 12(b) is an experimental results of $\hat{T}_{L}$, and Fig. 12(c) is the actual load torque value $T_{L}$ that utilize oscilloscope. From the view, can find that, the observation results can converged to reference value rapidly and have small overshoot when abrupt change of the load torque.

\subsection{Uncertainty in the electrical parameters}

To verify the robustness of a PMSM control system using the proposed PFC + MOO control scheme, the armature resistance, stator inductance and rotor-flux linkage, were set incorrectly in the controller. In comparison with the real values, the value of these three electrical parameters were respectively increased by $30 \%$, decreased by $30 \%$ and decreased by $20 \%$. Fig. 13 shows response curves in

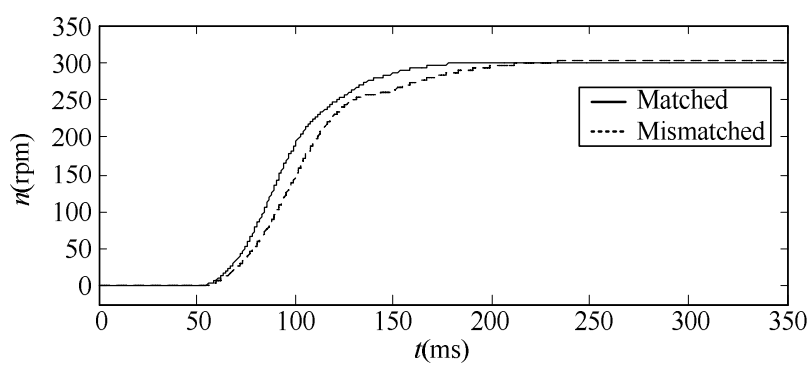

(a)

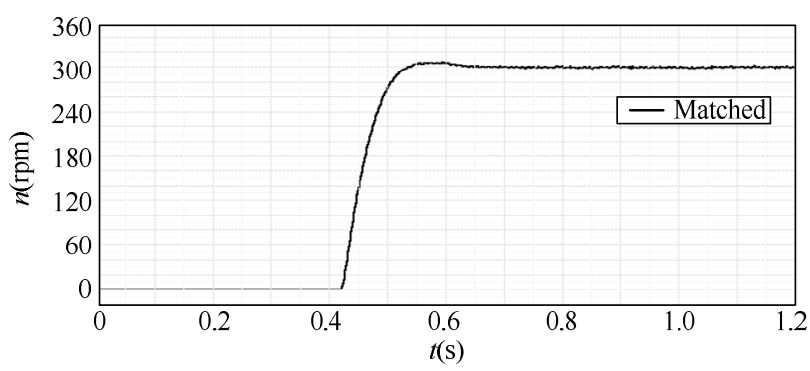

(b)

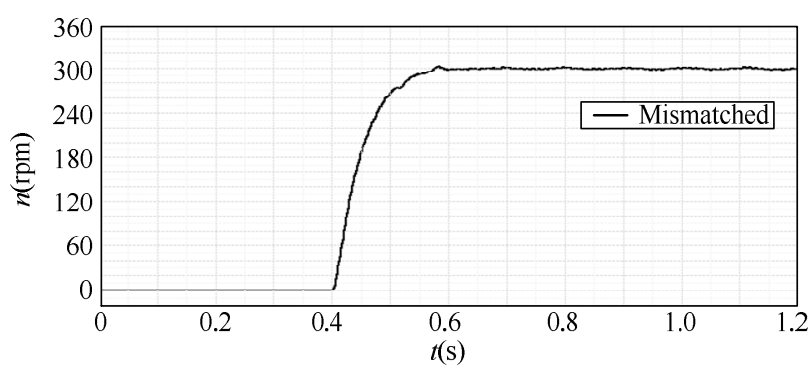

(c)

Fig. 13. Response curves in case of $300 \mathrm{rpm}$ speed trajectory tracking under uncertainty in the electrical parameters: (a) (Simulation); (b) Matched (Experimental); (c) Mismatched (Experimental). case of $300 \mathrm{rpm}$ speed trajectory tracking under matched and mismatched in the electrical parameters.

In comparison with the matched and mismatched mechanical parameters simulation response curves of Fig. 13(a), and experimental response curves of Fig. 13(b), Fig. 13(c). When PMSM electrical parameters mismatched, the speed setting time is more than parameters matched. However, the whole control system still be able to remain steady-state, it has good dynamic response process, the motor speed can be seen to converge to 300rpm under the presented control scheme. Therefore, the PMSM control systems can performance a stronger robustness of the proposed method against the inaccurate electrical parameters.

\subsection{Trajectory tracking performance evaluation under abrupt change of the load torque}

This test was performed to verify the performance of the proposed control strategy in terms of its disturbance rejection capability. The speed and current response waveforms comparison results are shown in Figs. 14-17 when adding an abrupt pulse shape $0.5 \mathrm{~N} \cdot \mathrm{m}$ load.

From the simulation Fig. 14 and Fig. 15, can be clearly seen that, after the load torque compensation, the $\mathrm{PFC}+\mathrm{MOO}$ scheme has less speed change than PFC scheme, it has been demonstrate that MOO can improve the disturbance rejection ability of PFC. Comparing the experimental Fig. 16(a) and Fig. 16(b), it can find that after adding observation torque feedback into PFC controller, speed decline is suppressed during the process of load

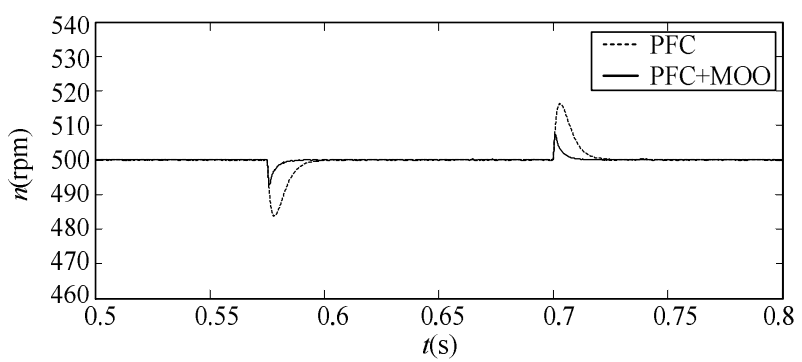

(a)

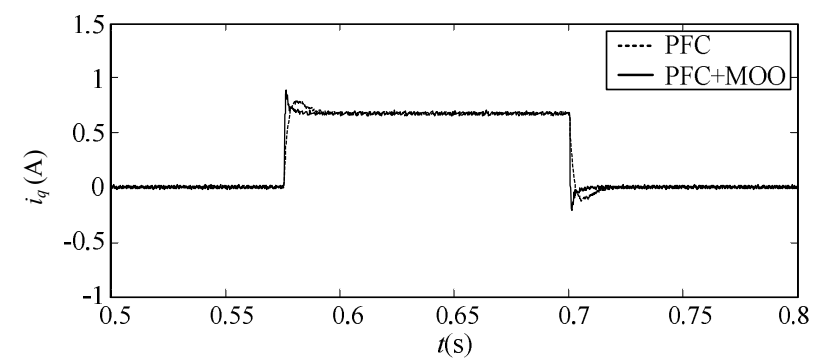

(b)

Fig. 14 Simulation response curves in case of $500 \mathrm{rpm}$ speed trajectory tracking under a sudden load torque disturbance. a Speed (PFC and PFC + $\mathrm{MOO}) . \mathrm{b} \mathrm{i}_{\mathrm{q}}$ (PFC and PFC + MOO $)$. 


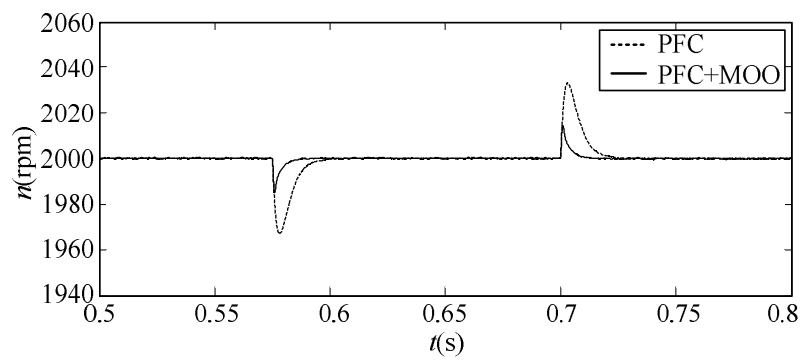

(a)

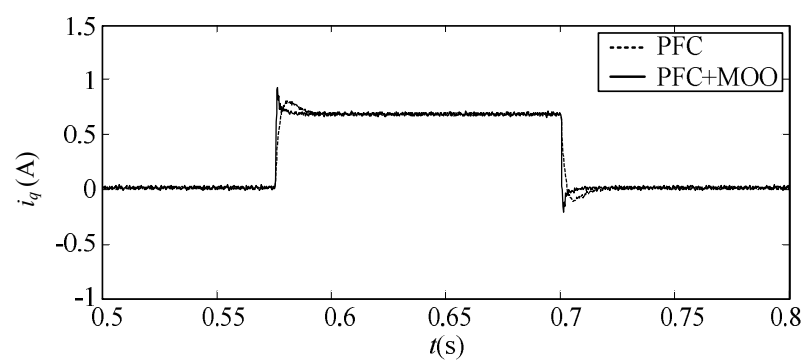

(b)

Fig. 15. Simulation response curves in case of $2000 \mathrm{rpm}$ speed trajectory tracking under a sudden load torque disturbance: (a) Speed (PFC and PFC + $\mathrm{MOO}$ ); (b) $\mathrm{i}_{\mathrm{q}}$ (PFC and PFC $\left.+\mathrm{MOO}\right)$.

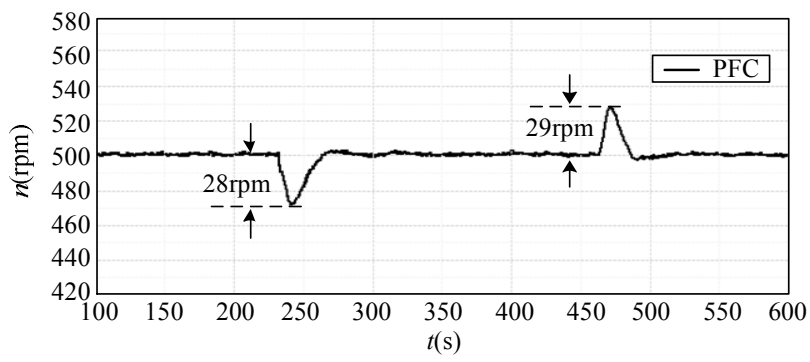

(a)

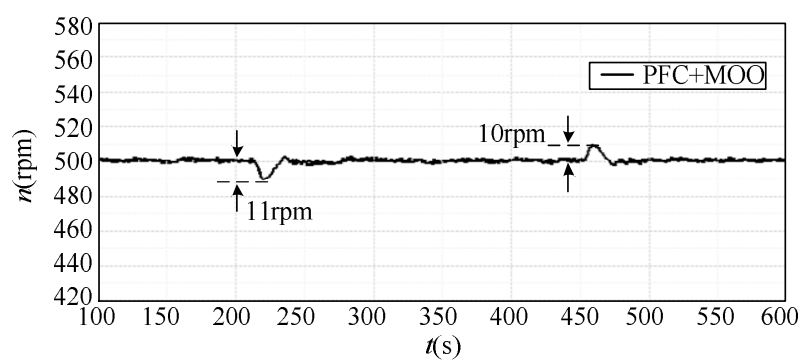

(b)

Fig. 16. Experimental response curves in case of $500 \mathrm{rpm}$ speed trajectory tracking under a sudden load torque disturbance. (a) Speed (PFC). (b) Speed $(\mathrm{PFC}+\mathrm{MOO})$.

changes, from $28 \mathrm{rpm}$ to $11 \mathrm{rpm}$, speed rise from $29 \mathrm{rpm}$ to 10rpm. Similarly, as shown in Fig. 17(a) and Fig. 17(b), speed decline and speed rise respectively from, $41 \mathrm{rpm}$ to 20rpm, and 40rpm to 19rpm. Fig. 14(b) and Fig. 15(b) shows waveforms of the $q$-axis current $i_{q}$. According to Lyapunov stability, the designed system is stable.

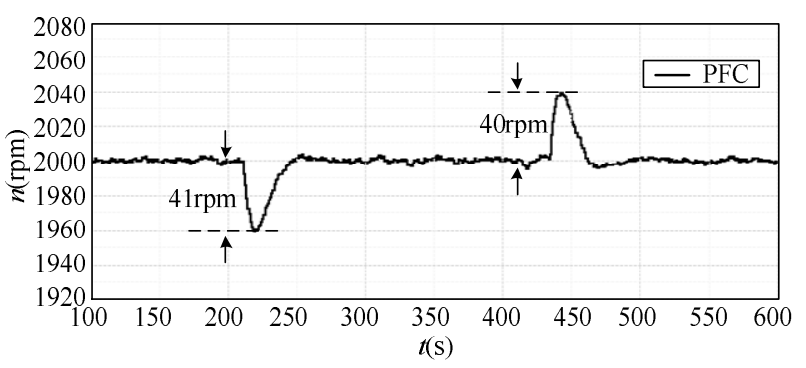

(a)

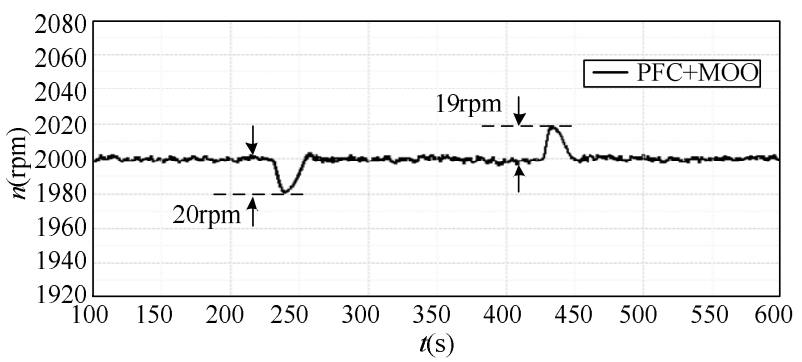

(b)

Fig. 17 Experimental response curves in case of $2000 \mathrm{rpm}$ speed trajectory tracking under a sudden load torque disturbance: (a) Speed (PFC); (b) Speed $(\mathrm{PFC}+\mathrm{MOO})$.

Therefore, compared with the PFC method, the proposed $\mathrm{PFC}+\mathrm{MOO}$ method has a less speed decrease and a shorter recovery time while maintaining a good dynamic performance, the steady-state error is quickly removed.

Remark 2: OS is the overshoot, $t_{s}$ is the setting time, the tolerance band of $t_{S}$ is selected as $\pm 2 \%$.

\section{Conclusions}

In this paper, we have presented a modified PFC strategy that using minimum-order observer to enhance prediction model precision. The role of the minimum-order observer is to estimated value of load torque disturbances, embedding disturbance information into the prediction model, thereby reconstruct the PFC regulator. Owing to this arrangement, the predicted output error in the PFC algorithm is reduced, and the tracking performance is improved. Moreover, the structure of minimum-order observer is very simple and can be realized at a very low computational load. The simulation and experimental results show that the proposed control method can effectively strengthen the system dynamic control performance and disturbance rejection.

\section{Acknowledgments}

The authors would like to thank the support from National 863 Project of China High-Tech Development Plan (Project No. 2011AA04A105). 


\section{References}

[1] Line, C., Manzie, C., Good, M.C.: 'Electromechanical brake modeling and control: From PI to MPC', IEEE Trans. Control Syst. Technol., 2008, 16, (3), pp. 446457.

[2] Xia, C.L., Ji, B.N., Yan, Y.: 'Smooth speed control for low-speed high-torque permanent-magnet synchronous motor using proportional-integral-resonant controllers', IEEE Trans. Ind. Electron., 2015, 62, (4), pp. 2123-2134.

[3] Tiwari, A.N., Agarwal, P., Srivastava, S.P.: 'Performance investigation of modified hysteresis current controller with the permanent magnet synchronous motor drive', IET Electr. Power Appl., 2010, 4, (2), pp. 101-108.

[4] Jung, J.W., Leu, V.Q., Do, T.D., Kim, E.K., Choi, H.H.: 'Adaptive PID speed control design for permanent magnet synchronous motor drives', IEEE Trans. Ind. Electron., 2015, 30, (2), pp. 900-908.

[5] Lin, F.J., Yang, K.J., Sun, I.F., Chang, J.K.: 'Intelligent position control of permanent magnet synchronous motor using recurrent fuzzy neural cerebellar model articulation network', IET Electr. Power Appl., 2015, 9, (3), pp. 248-264.

[6] Bernardes, T., Montagner, V.F., Gründling, H.A., Pinheiro, H.: 'Discrete-time sliding mode observer for sensorless vector control of permanent magnet synchronous machine', IEEE Trans. Ind. Electron., 2014, 61, (4), pp. 1679-1691.

[7] Errouissi, R., Ouhrouche, M., Chen, W.H., Trzynadlowski, A.M.: 'Robust nonlinear predictive controller for permanent-magnet synchronous motors with an optimized cost function', IEEE Trans. Ind. Electron., 2012, 59, (7), pp. 2849-2858.

[8] Arashloo, R. S., Romeral, M. J. L., Salehifar, M., Eguilaz, M. M.: 'Genetic algorithm-based output power optimisation of fault tolerant five-phase brushless direct current drives applicable for electrical and hybrid electrical vehicles'. IET Electr. Power Appl., 2014, 8, (7), pp. 267-277.

[9] Lin, F. J., Hung, Y. C., Hwang, J. C., Chang, I. P., Tsailet, M.T.: 'Digital signal processor-based probabilistic fuzzy neural network control of in-wheel motor drive for light electric vehicle', IET Electr. Power Appl., 2012, 6, (2), pp. 47-61.

[10] Kim, W., Shin, D., Chung, C. C., 'Microstepping using a disturbance observer and a variable structure controller for permanent-magnet stepper motors', IEEE Trans. Ind. Electron., 2013, 60, (7), pp. 26892699.

[11] Zhang, X.G., Sun, L.Z., Zhao, K., and Sun, L.: 'Nonlinear speed control for PMSM system using sliding-mode control and disturbance compensation techniques', IEEE Trans. Ind. Electron., 2013, 28, (3), pp. 1358-1365.
[12] Wu, S., Wang, Y., Cheng, S.: 'Optimal reset control design for current control and uncertainties estimation in permanent magnet synchronous', IET Electr. Power Appl., 2012, 6, (2), pp. 122-132.

[13] Garcia, C. E., Prett, D. M., Morari, M.: 'Model predictive control: theory and practice - a survey', Automatica, 1989, 25, (3), pp. 335-348.

[14] Chen, W. H., Ballance, D. J., Gawthrop, P. J.: 'Optimal control of nonlinear systems: a predictive control approach', Automatica, 2003, 39, (4), pp. 633-641.

[15] Errouissi, R., Ouhrouche, M., Chen, W.H., Trzynadlowski, A.M.: 'Robust cascaded nonlinear predictive control of a PMSM with anti-windup compensator', IEEE Trans. Ind. Electron., 2012, 59, (8), pp. 30783088.

[16] Chen, Y., Liu, T. H., Hsiao, C. F., Lin, C. K.: 'Implementation of adaptive inverse controller for an interior permanent magnet synchronous motor adjustable speed drive system based on predictive current control', IET Electr. Power Appl., 2015, 9, (1), pp. 60-70.

[17] Chai, S., Wang, L.P., Rogers, E.: 'A cascade MPC control structure for a PMSM with speed ripple minimization," IEEE Trans. Ind. Electron., 2013, 60, (8), pp. 2978-2987.

[18] Preindl, M., Bolognani, S.: 'Model predictive direct speed control with finite control set of PMSM drive systems,' IEEE Trans. Ind. Electron., 2013, 28, (2), pp. 1007-1015.

[19] Kuntze, H.B., Jacubasch, A., Richalet, J. Arber, C.: 'On the predictive functional control of an elastic industrial robot', in Proc. IEEE Conf. Decision and Control, Athens, 1986, 3, pp. 1877-1881.

[20] Richalet, J., Abu, E., Arber, C., Kuntze, H. B., Jacubasch, A., Schill, W.: 'Predictive functional control: application to fast and accurate robots', in Proc. 10th IFAC Worl. Congr., Munich, 1987, 4, pp. 251258.

[21] Richalet, J. 'Industrial applications of model based predictive control', Automatica, 1993, 29, (5), pp. 1251-1274.

[22] Hassan, A.A. Kassem, A.M.: 'Modeling, simulation and performance improvements of a PMSM based on functional model predictive control', Arab. J. Sci. Eng., 2013, 38, (11), pp. 3071-3079.

[23] Liu, H.X., Li, S.H.: 'Speed control for PMSM servo system using predictive functional control and extended state observe', IEEE Trans. Ind. Electron., 2012, 59, (2), pp. 1171-1183.

[24] Leu, V.Q., Choi, H.H., Jung, J. W., 'Fuzzy sliding mode speed controller for PM synchronous motors with a load torque observer', IEEE Trans. Power Electron., 2012, 27, (3), pp. 1530-1539.

[25] Pongam, S., Sangwongwanich, S.: 'Stability and dynamic performance improvement of adaptive full- 
order observers for sensorless PMSM drive', IEEE Trans. Power Electron., 2012, 27, (2), pp. 588-600.

[26] Chen, B., Yao, W., Chen, F., Lu, Z.: 'Parameter sensitivity in sensorless induction motor drives with the adaptive full-order observer', IEEE Trans. Ind. Electron., 2015, 62, (7), pp. 4307-4318.

[27] Luenberger, D. G.: 'Observing the state of a linear system', IEEE Trans. Mil. Electron., 1964, 8, (2), pp. 74-80.

[28] Luenberger, D.G.: 'An introduction to observers', IEEE Trans. Autom. Control, 1971, 16, (6), pp. 596602.

[29] Hafez, B., Abdel-Khalik, A. S., Massoud, A. M., Ahmed, S., Lorenz, R.D.: 'Single-sensor-based threephase permanent-magnet synchronous motor drive system with luenberger observers for motor line current reconstruction', IEEE Trans. Ind. Appl., 2014, 50, (4), pp. 2602-2613.

[30] Preindl, M., Schaltz, E., 'Sensorless model predictive direct current control using novel second-order PLL observer for PMSM drive systems', IEEE Trans. Ind. Electron., 2011, 58, (9), pp. 4087-4095.

[31] Vu, N. T. T., Choi, H. H., Kim, R. Y., Jung, J. W.: 'Robust speed control method for permanent magnet synchronous motor'. IET Electr. Power Appl., 2012, 6, (7), pp. 399-411.

[32] Chan, T. F, Wang, W., Borsje, P., Wong, Y. K., Ho, S.L.: 'Sensorless permanent-magnet synchronous motor drive using a reduced-order rotor flux observer', IET Electr. Power Appl., 2008, 2, (2), pp. 88-98.

[33] Grzesiak, L. M, Tarczewski, T.: 'PMSM servo-drive control system with a state feedback and a load torque feedforward compensation'. COMPEL-The international journal for computation and mathematics in electrical and electronic engineering, 2012, 32, (1), pp. 364-382.

[34] Fernando, T.L., Trinh, H., Jennings, L.: 'Functional observability and the design of minimum order linear functional observers', IEEE Trans. Autom. Control., 2010, 55, (5), pp. 1268-1273.

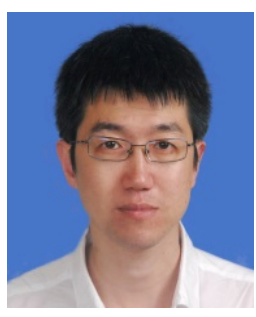

Shuang Wang He received his B.S., M.S. and Ph.D. degrees in Electrical Engineering from Harbin Institute of Technology, Harbin, China, in 2000, 2005 and 2009, respectively. Since 2010, he has been with the School of Mechatronic Engineering and Automation, Shanghai University, Shanghai, China, where he is presently working as Assistant Professor. His current research interests include intelligent control theory and its application to new energy vehicles, power electronics and servo control systems.

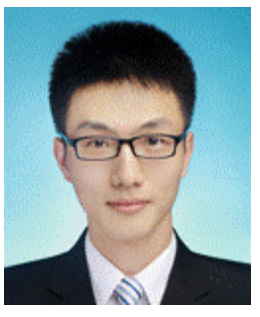

Junyong Fu He received his B.S. degree in Electrical Engineering from Nantong University, Nan Tong, China, in 2010 . He is presently a postgraduate student at Shanghai University, Shanghai, China. His current research interests include new energy vehicles, control theory and its application to electric drive servo systems

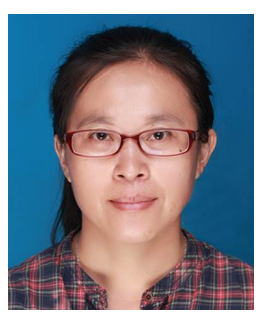

Ying Yang She received Ph.D. degrees in vehicle operation engineering from Tongji University, Shanghai, China, in 2007. Since 2007, she has been with the School of Mechatronic Engineering and Automation, Shanghai University, Shanghai, China, where she is presently working as Associate Professor. Her current research interests include control of new energy vehicles and servo control systems.

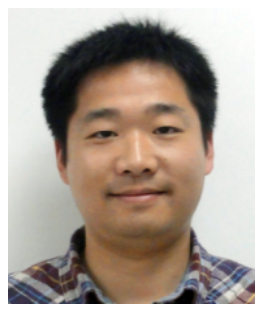

Jian Shi He received his B.S. degree from Xi'an University of Technology, Xi'an, China, his M.S. degree from Fuzhou University, Fuzhou, China, and his Ph.D. degree from Harbin Institute of Technology, Harbin, China, in 2004, 2007 and 2013 respectively. From 2007 to 2010, he was with ThyssenKrupp Elevator Co, China. Since 2014, he has been with the School of Mechatronic Engineering and Automation, Shanghai University, Shanghai, China, where he is currently an assistant professor. His research interests include electric machines, power electronics, and control systems. 